\title{
Treatment of postherpetic neuralgia: focus on pregabalin
}

\author{
Kimberly A Cappuzzo \\ The Geriatric Pharmacotherapy \\ Program, Virginia Commonwealth \\ University, School of Pharmacy, \\ Richmond, Virginia, USA
}

\begin{abstract}
Postherpetic neuralgia (PHN) is a devastating, chronic pain syndrome that can develop following an outbreak of herpes zoster and becomes increasingly common as patients age. PHN can be difficult to treat and often requires trials of multiple agents to achieve significant pain relief. Pregabalin is the newest agent to gain approval for PHN. Data suggest efficacy for relief of pain and sleep disturbance secondary to PHN in affected patients. Although there are no head-to-head comparisons, pregabalin appears comparable to gabapentin and other first-line agents for treating PHN.
\end{abstract}

Keywords: pregabalin, postherpetic neuralgia, neuropathic pain

\section{Introduction}

Herpes zoster (HZ) or shingles is a result of reactivation of the varicella zoster virus (VZV), the same virus that causes chickenpox. After the acute varicella infection (ie, chickenpox in childhood), the virus can lie dormant for decades in sensory nerve ganglia, usually involving the lumbar, thoracic and trigeminal ganglia. HZ results when dormant VZV in these nerves is reactivated, possibly secondary to a decline in specific cell-mediated immunity to VZV with aging and/or immunosuppression. The majority of cases of $\mathrm{HZ}$ are seen in older adults, with incidence increasing sharply at around 50 years of age and doubling by age $80 .{ }^{1,2}$ Neither gender, race, nor ethnicity appear to be risk factors for HZ. ${ }^{3}$

A patient with HZ may first develop a prodromal syndrome with fever, malaise, and pain that is generally localized to the affected dermatome. This pain may include burning, itching, and hypersensitivity and may precede the rash by 4 to 5 days., Because many patients initially present with severe pain as their chief complaint, patients are often misdiagnosed.

Pain is the major symptom of HZ, and, in most patients, the pain resolves spontaneously over time. However, about $10 \%$ to $15 \%$ of patients develop chronic, debilitating postherpetic pain that persists after the characteristic rash disappears ${ }^{2,5}$ with some patients experiencing persistent pain for years. ${ }^{6}$ Posterherpetic neuralgia (PHN) is defined in the literature as pain that persists for more than 1 to 6 months after rash resolution. In most clinical trials, $\mathrm{PHN}$ is defined as pain persisting for more than 3 months after resolution of the rash. ${ }^{2,3,5}$

PHN is often characterized by a combination of throbbing or burning pain, intermittent sharp pains, altered sensory perception, including paresthesia, and allodynia (painful response to an innocuous stimulus). ${ }^{3}$ The pain may extend beyond the borders of the original zoster rash. The variety of symptoms likely result from injury to the dorsal root ganglia and dorsal horn as well as injury to the peripheral nerves. The incidence, duration, and severity are all related to increasing age; in fact, PHN is uncommon in those less than 60 years of age. ${ }^{1-4}$ Presence of pain prior to rash eruption, 
rash severity, and inflammation and fever are thought to all have an effect on PHN severity. ${ }^{3} \mathrm{PHN}$ pain is often severe, unrelenting, and exhausting. As a result, PHN can dramatically affect a patient's quality of life and functional status. Patients may develop insomnia, weight loss, chronic fatigue, and an inability to perform daily activities. It is estimated that more than $50 \%$ of patients with PHN have sleep disturbances, and about $25 \%$ report a decrease in socialization. Eventually a patient with PHN may lose the ability for self-care, leading to depression and social isolation. ${ }^{1,2,7,8}$

Successful management of PHN can be complicated and challenging, especially with the fact that there is no definitive treatment algorithm specifically for patients with PHN. In recent years, there have been a number of published guidelines proposed for the treatment of neuropathic pain in general. ${ }^{5,9-12}$ These recommendations are essentially based on evidence of efficacy from randomized controlled trials (RCTs) of pharmacologic therapies; there is a lack of clinical trials directly comparing efficacy and safety of one pharmacotherapy versus another. ${ }^{12,13}$ These guidelines uniformly recommend tricyclic antidepressants (TCAs), opioids, and anticonvulsants as first-line therapeutic options for treating neuropathic pain. First or second-line recommendations include topical treatments (eg, topical lidocaine), depending on the source.

Both gabapentin and pregabalin (PGB) are oral anticonvulsants approved for the management of PHN. They are both recommended as first-line therapeutic choices for neuropathic pain based on several RCTs., ${ }^{5,-12}$ Although there have been no head-to-head RCTs between these 2 agents in patients with $\mathrm{PHN}$, both have significantly reduced pain $(\mathrm{p}<0.01)$ and improved sleep $(\mathrm{p}<0.01)$. $^{7,15-18}$

This review will focus specifically on PGB, the newest agent to gain approval for PHN. A general overview of the drug will be given, followed by a review of clinical trial efficacy and adverse effects data in PHN patients, concluding with a discussion of PGB's place in therapy in treating PHN.

\section{Pregabalin overview: pharmacology and pharmacokinetics}

PGB was first approved in the European Union by the European Agency for the Evaluation of Medicinal Products for the treatment of peripheral neuropathic pain in July 2004. PGB received conditional approval by the US Food and Drug Administration for the treatment of diabetic peripheral neuropathy (DPN) and PHN in December 2004 and was granted final approval after controlled substance scheduling by the US Drug Enforcement Agency in August 2005. ${ }^{19}$ PGB is a schedule V controlled substance based on a study with 15 recreational sedative/hypnotic drug users who rated the "desirability" of a single dose of PGB $450 \mathrm{mg}$ as similar to a single dose of diazepam $30 \mathrm{mg} \cdot{ }^{19-21}$ Indeed, euphoria was reported in 4\% of PGB-treated patients versus $1 \%$ of placebo-treated patients in over 5500 patients in controlled clinical trials, and withdrawal symptoms suggestive of physical dependence were reported upon abrupt discontinuation of PGB in some studies. ${ }^{20}$

The exact mechanism of action of PGB is unclear but is thought to be similar to that of gabapentin. PGB is a structural analogue of GABA, but it is inactive at GABA-A or -B receptors, it is not converted into GABA or a GABA antagonist, and it does not affect GABA uptake. ${ }^{7,21,22}$ PGB is an alpha2-delta ligand. PGB may alter the release of several neurotransmitters, by selectively binding to the alpha2-delta auxiliary protein subunit of voltage-gated calcium channels. By tightly binding to alpha2-delta protein, PGB reduces the influx of calcium, thereby reducing the release of neurotransmitters, including glutamate, norepinephrine, and substance $P$. These mechanisms are thought to result in the anticonvulsant, anxiolytic, and analgesic properties exhibited by PGB.

PGB was created in an attempt to develop a compound that would retain the biologic activity of gabapentin while improving its pharmacokinetic profile. PGB is well absorbed following oral administration with an oral bioavailability of $\geq 90 \%$. The bioavailability of gabapentin actually decreases from $60 \%$ to $27 \%$ with increasing doses due to saturable absorption (900 mg/day versus $4800 \mathrm{mg} /$ day, respectively). ${ }^{20,21,23}$ Unlike gabapentin, PGB exhibits linear pharmacokinetics. Elimination of PGB is virtually proportional to creatinine clearance $(\mathrm{CrCl})$. Dosage adjustments for renal dysfunction are provided (Table 1). Ninety-eight percent of a dose of PGB is excreted unchanged in the urine, and the drug undergoes negligible metabolism with no anticipated CYP 450 enzyme drug interactions. PGB pharmacokinetics do not appear to be affected by race or gender and have not been studied in pediatric patients. ${ }^{20,21,24}$ Renal clearance of PGB appears to decrease with age, consistent with age-related changes in renal function. Hence, older adults often require renal adjustment of $\mathrm{PGB}$ dosing. ${ }^{20} \mathrm{PGB}$ is effectively cleared by hemodialysis; $50 \%-60 \%$ is removed from the circulation following a 4-hour hemodialysis session. Therefore, a supplemental dose should be given immediately following every 4-hour hemodialysis treatment (Table 1). ${ }^{20,21,24}$

\section{Literature review: pregabalin for PHN}

The efficacy of PGB for management of PHN has been demonstrated in 3 double-blind, placebo-controlled, multicenter 
Table I Dosage adjustment of pregabalin based on renal clearance ${ }^{20}$

\begin{tabular}{llllll}
\hline Creatinine clearance $(\mathbf{m l} / \mathbf{m i n})$ & \multicolumn{2}{l}{ Total daily dose of pregabalin $(\mathbf{m g} / \mathbf{d a y})$} & Total daily dose to be divided \\
\hline$\geq 60$ & 150 & 300 & 450 & 600 & $2-3$ times daily \\
$30-59$ & 75 & 150 & 225 & 300 & $2-3$ times daily \\
$15-29$ & $25-50$ & 75 & $100-150$ & 150 & Once or twice daily \\
$<15$ & 25 & $25-50$ & $50-75$ & 75 & Once daily \\
Supplemental dose after $\mathrm{HD}^{\mathrm{a}}$ & $25-50$ & $50-75$ & $75-100$ & $100-150$ & \\
\hline
\end{tabular}

a Supplemental dose after hemodialysis is a single dose to be administered after each 4-hour hemodialysis session.

Abbreviation: HD, hemodialysis.

clinical trials involving patients with PHN, and 1 randomized, double-blind, placebo-controlled study in patients with chronic neuropathic pain including PHN. ${ }^{7,14,18,25}$ Studies comparing PGB with other agents used to treat PHN are not available. Patients failing to respond to gabapentin were excluded from most of these studies. ${ }^{7,14,18}$ An important point to note: all 4 trials described herein were sponsored by the manufacturer of PGB (Pfizer), and the trials were conducted at least in part by Pfizer employees.

An 8-week trial was conducted in 173 patients with PHN, defined as pain persisting for $>3$ months following the healing of a $\mathrm{HZ}$ rash. ${ }^{14}$ Concomitant medications that were allowed at stable doses during the trial included opioid and non-opioid analgesics, NSAIDs, aspirin, acetaminophen ( $\leq 4 \mathrm{~g} /$ day), and antidepressants including SSRIs. Benzodiazepines, skeletal muscle relaxants, oral steroids, local and topical agents for PHN, and anticonvulsants were prohibited. Patients were randomized to either PGB $(n=89)$ or placebo $(n=84)$. Doses were given three times a day. Those on PGB received either $600 \mathrm{mg} /$ day $(\mathrm{CrCl}>60 \mathrm{~mL} / \mathrm{min})$ or $300 \mathrm{mg} /$ day $(\mathrm{CrCl} 30-60 \mathrm{~mL} / \mathrm{min})$. This renal dosage adjustment is based on pharmacokinetic studies that demonstrated comparable steady-state concentrations of PGB, thus allowing these 2 subsets to be combined as a single PGB treatment arm for the purpose of pain relief assessment. ${ }^{14,15}$ Mean age of the patients was 71.5 years, with $82 \%$ of patients over 65 years of age, and the mean duration of PHN was 33.8 months. Patients were required to have an average daily pain score of at least 4 on an 11-point numerical rating scale $(0=$ no pain; $10=$ worst possible pain $)$ during the 7-day baseline period. Additionally, patients were required to have a pain score of $\geq 40 \mathrm{~mm}$ on the $100 \mathrm{~mm}$ visual analog scale (VAS) of the Short-Form McGill Pain Questionnaire (SF-MPQ) at baseline and at randomization. The primary efficacy point was the mean of the last 7 daily pain scores on the 11-point pain scale. Patients treated with PGB had greater reductions in mean pain scores than those treated with placebo ( 3.60 versus $5.29 ; \mathrm{p}<0.0001$ ). A decrease in pain was seen in PGB-treated patients as early as day 1 and maintained throughout the study. In addition, significantly more patients receiving PGB had $\geq 50 \%$ reduction in mean pain scores than those receiving placebo. As a secondary endpoint, daily sleep interference scores were measured for all study participants by assessing the degree to which pain interfered with sleep during the previous 24 hours on an 11-point numerical rating scale. A score of 0 indicates pain did not interfere with sleep, and a score of 10 indicates pain completely interfered with sleep. Sleep interference scores were recorded in subjects' daily diaries upon awakening. Significant improvements in mean sleep interference scores (derived from subjects' last 7 days of diary entries while receiving study drug) were observed. At study end, the mean sleep interference score was 1.93 in PGB-treated patients versus 3.51 in placebo-treated subjects $(\mathrm{p}=0.0001) .{ }^{14}$

A second 8-week trial was conducted in 238 patients with PHN of $>6$ months in duration after the healing of the HZ rash. ${ }^{7}$ Concomitant medications that were allowed at stable doses during the study period included NSAIDs, acetaminophen, opioid and non-opioid analgesics, and antidepressants. Anticonvulsants and benzodiazepines had to be discontinued at least 14 days prior to receiving study medication. Patients with a $\mathrm{CrCl} \leq 30 \mathrm{~mL} / \mathrm{min}$ were excluded from the trial. Patients were randomized to either PGB $150 \mathrm{mg} /$ day $(\mathrm{n}=81)$, PGB $300 \mathrm{mg} /$ day $(\mathrm{n}=76)$, or placebo $(n=81)$. Doses were given three times a day. Mean age of the patients was similar to those in the study by Dworkin and colleagues (73.2 years, 71.3, and 71.9 years for the placebo, $150 \mathrm{mg} /$ day, and $300 \mathrm{mg} /$ day groups, respectively), and the mean duration of PHN ranged from 44.8 to 40.7 months for the placebo, $150 \mathrm{mg} /$ day, and $300 \mathrm{mg} /$ day groups respectively. Patients were required to have an average daily pain score of at least 4 on an 11-point numerical rating scale $(0=$ no pain; $10=$ worst possible pain $)$ during the 7 -day baseline period. In addition, patients were required to have a score of $\geq 40 \mathrm{~mm}$ on the $100 \mathrm{~mm}$ VAS of the SF-MPQ at 
baseline and at randomization. The primary efficacy point was the mean of the last 7 daily pain scores on the 11-point pain scale. Endpoint mean pain scores were significantly reduced for both PGB $150 \mathrm{mg} /$ day and PGB $300 \mathrm{mg} /$ day groups compared to placebo. Significant improvement in pain was noted as early as week 1 and maintained throughout the study. There appeared to be some additional pain relief from the $300 \mathrm{mg}$ /day dose versus the $150 \mathrm{mg} /$ day dose per the endpoint mean pain scores reported by the patients in each PGB group. Those receiving $300 \mathrm{mg} /$ day of PGB reported a mean pain score of 4.76 as compared to a 5.14 mean pain score in those receiving $150 \mathrm{mg} /$ day; patients on placebo reported a mean pain score of $6.33(\mathrm{p}=0.0001$ and 0.0002 for $300 \mathrm{mg} /$ day and $150 \mathrm{mg} /$ day versus placebo, respectively). A significantly larger proportion of patients in both treatment groups obtained a $\geq 50 \%$ reduction in mean pain score from baseline to study end point $(26 \%$ on $150 \mathrm{mg} /$ day [ $=0.006]$ and $28 \%$ on $300 \mathrm{mg} /$ day $[\mathrm{p}=0.003]$ versus $10 \%$ on placebo). As with the previous trial, daily sleep interference scores were recorded by study subjects in diaries using an 11-point numerical rating scale. Both doses of PGB significantly reduced weekly mean sleep interference scores versus placebo (3.13 for those on $150 \mathrm{mg} /$ day [p $=0.0003], 2.81$ for those on $300 \mathrm{mg} /$ day $[\mathrm{p}=0.0001]$ and 4.24 for those on placebo). ${ }^{7}$

A 13-week trial in 370 patients with $\mathrm{PHN}$ of $\geq 3$ months' duration following the healing of $\mathrm{HZ}$ lesions was conducted to evaluate the efficacy of twice daily PGB therapy. ${ }^{18}$ Stable medication regimens (defined as medications taken $\geq 30$ days prior to study entry) of non-opioid analgesics (eg, paracetamol, noramidopyrine), opioids, anti-inflammatory medications, and antidepressants were allowed during the trial. Prohibited medications included long-acting benzodiazepines, skeletal muscle relaxants, steroids, and anticonvulsants, among others; these had to be discontinued at least 7 days prior to study entry. Patients with a $\mathrm{CrCl} \leq 30 \mathrm{~mL} / \mathrm{min}$ were excluded from the trial. Patients were randomized to 1 of 4 treatment groups: placebo $(\mathrm{n}=93), 150 \mathrm{mg} /$ day $(\mathrm{n}=87), 300 \mathrm{mg} /$ day $(\mathrm{n}=98)$, and $600 \mathrm{mg} /$ day $(\mathrm{n}=90)$. Doses were divided twice daily. Because a $50 \%$ reduction in $\mathrm{CrCl}$ is expected to result in a doubling of PGB exposure, patients in the $600 \mathrm{mg} /$ day group were stratified based on $\mathrm{CrCl}$ : patients with $\mathrm{CrCl}>60 \mathrm{~mL} / \mathrm{min}$ received $600 \mathrm{mg} / \mathrm{day}$, and those patients with $\mathrm{CrCl}>30$ and $\leq 60 \mathrm{~mL} / \mathrm{min}$ received $300 \mathrm{mg} /$ day, a dosage believed to provide equivalent exposure to $600 \mathrm{mg}$ /day in patients with $\mathrm{CrCl}>60 \mathrm{~mL} / \mathrm{min}$, based on pharmacokinetic studies. ${ }^{18,26}$ Mean age of the patients was 70.7 years, with $82 \%$ of patients over 65 years of age, and the mean duration of PHN was
40.7 months. Patients were required to have an average daily pain score of at least 4 on an 11-point numerical rating scale ( $0=$ no pain; $10=$ worst possible pain) on at least 4 days during the 7-day baseline period. Additionally, patients were required to have a score of $\geq 40 \mathrm{~mm}$ on a $100 \mathrm{~mm}$ VAS at baseline and at randomization. Primary outcome measure was the endpoint mean pain score from the last 7 days of the patients' daily pain diaries. As with the previous two trials discussed, this study assessed related sleep interference due to pain as a secondary outcome. Daily sleep interference scores were again recorded by study subjects in diaries using an 11-point numerical rating scale. At endpoint, $\mathrm{PGB}$ demonstrated significant dose-dependent improvement in mean pain scores and significant improvement in mean sleep interference versus placebo. Mean pain scores decreased in a dose-dependent fashion as follows: PGB $150 \mathrm{mg}$ /day was 5.26, PGB $300 \mathrm{mg} /$ day 5.07, PGB $600 \mathrm{mg} /$ day 4.35, and these were all significantly lower versus the placebo mean pain score of $6.14(\mathrm{p}=0.0077,0.0008$, and 0.0001 , for PGB 150, 300, and $600 \mathrm{mg}$ /day, respectively). Mean sleep interference scores showed a similar dosedependent pattern: PGB $150 \mathrm{mg} /$ day 3.07, PGB $300 \mathrm{mg} /$ day 2.84, and PGB $600 \mathrm{mg} /$ day 2.17, and these were all significantly lower than the placebo score of $4.10(\mathrm{p}=0.0007,0.0001$, and 0.0001 , for PGB 150, 300, and $600 \mathrm{mg} /$ day, respectively). These improvements in pain and sleep were seen as early as Week 1 of treatment. Additionally, significantly more patients in the PGB-treated groups demonstrated a $\geq 50 \%$ reduction in pain from baseline than those on placebo $(26.4 \%, 26.5 \%$, $37.5 \%$, and $7.5 \%$ in the PGB $150 \mathrm{mg} /$ day, $300 \mathrm{mg} /$ day, $300 / 600 \mathrm{mg} /$ day, and placebo groups, respectively; $\mathrm{p}=0.001$ for each PGB group versus placebo). The number needed to treat (NNT) based on those with $\geq 50 \%$ reduction in pain from baseline for all PGB dosages combined was 4.4. ${ }^{18}$

A 12-week trial examined the efficacy and tolerability of flexible- and fixed-dose regimens of PGB versus placebo in patients with PHN and painful DPN. ${ }^{25}$ PHN patients had pain present for $\geq 3$ months after the healing of the $\mathrm{HZ}$ rash. Unlike the previously discussed trials, patients who had previously taken gabapentin were permitted in the trial, regardless of dose or duration of exposure. Use of SSRIs, aspirin, short-acting benzodiazepines (for insomnia), and paracetamol was permitted during the trial. Medications that were prohibited include drugs commonly used to treat neuropathic pain (eg, skeletal muscle relaxants, capsaicin cream, opioids, benzodiazepines), anticonvulsants, non-SSRI antidepressants, and drugs that may cause retinotoxicity (eg, hydroxychloroquine, thioridazine). Prohibited drugs had to be discontinued at least 7 days prior to the baseline visit. 
Patients with $\mathrm{CrCl}<60 \mathrm{~mL} / \mathrm{min}$ and those that had abused alcohol or illicit drugs within the past 2 years were excluded. Patients were randomized to placebo $(n=65)$, flexibledose PGB $(150-600 \mathrm{mg} /$ day; $\mathrm{n}=141)$, or fixed-dose PGB ( $600 \mathrm{mg} /$ day; $\mathrm{n}=132$ ). Patients randomized to the flexibledose group received weekly escalating doses (150, 300, 450 , and $600 \mathrm{mg} /$ day) of PGB based on response and tolerability. These patients were permitted a single downward dose titration during the first 4 weeks; if this occurred, the patient would remain on that dosage for the study duration. The flexible-dosing schedule was thought to more closely mimic actual clinical practice. Mean age of the patients was between 61 and 62 years of age, slightly younger than the other 3 trials. The mean duration of PHN was between 35 and 39 months. As was the case with the previous 3 trials, patients were required to have a score of $\geq 40 \mathrm{~mm}$ on a $100 \mathrm{~mm}$ VAS at baseline and at randomization. Primary outcome measure was the endpoint mean pain score from the last 7 days of the patients' daily pain diaries according to the 11-point numerical pain scale discussed previously. This study assessed related sleep interference due to pain as a secondary outcome using a sleep interference diary similar to the other trials discussed. Flexible-dose PGB significantly reduced mean endpoint pain scores versus placebo by Week 2 $(p=0.021)$ and throughout the study $(p \leq 0.013)$; fixed dose PGB significantly reduced mean endpoint pain scores versus placebo by the end of Week $1(p=0.007)$ and subsequently throughout the rest of the trial $(\mathrm{p}<0.001)$. The magnitude in reduction of pain symptoms was similar in patients with either PHN or DPN. Moreover, significantly more patients in the PGB-treated groups demonstrated a $\geq 50 \%$ reduction in pain from baseline than those on placebo $(48.2 \%$ in the flexible-dose PGB group, 52.3\% in the fixed-dose PGB group, and $24.2 \%$ in the placebo group; $p<0.001$ for each PGB group versus placebo). The NNT based on those with $\geq 50 \%$ reduction in pain from baseline for all PGB-treated patients combined was 3.8. Sleep interference scores improved significantly at endpoint over placebo for patients in each PGB treatment group ( $p<0.001$ for each group versus placebo; exact values not given). ${ }^{25}$

\section{Adverse effects/tolerability}

The most common adverse effects with PGB treatment noted in PHN trials were dizziness, somnolence, and peripheral edema. ${ }^{7,14,18,25}$ Other notable side effects commonly reported with PGB use include dry mouth, blurred vision, weight gain, ataxia, headache, "thinking abnormal," and nausea. Dizziness and somnolence were the most common adverse effects leading to study withdrawal. ${ }^{20}$ Dizziness and somnolence occurred more frequently at higher doses and began shortly after starting PGB ${ }^{20,21}$ Symptoms including insomnia, nausea, headache, and diarrhea were reported by some patients following abrupt withdrawal of PGB. Therefore, PGB should be tapered gradually over a minimum of 1 week rather than discontinued abruptly. Weight gain noted in clinical trials of PGB was not limited to patients with peripheral edema. Even though weight gain was related to dose and duration of exposure to PGB, it did not appear to be associated with gender, age, or baseline BMI. A higher incidence of weight gain and peripheral edema were noted in patients taking both PGB and a thiazolidinedione, compared with patients taking either agent alone. Hence, care should be taken when co-administering PGB and one of these agents. ${ }^{20,21}$ The package insert cautions about the use of PGB in heart failure patients with NYHA Class III or IV cardiac status, ${ }^{20}$ and there have been reports of heart failure decompensation in patients using PGB for neuropathic pain. ${ }^{27}$

\section{Place in therapy}

As discussed previously, PGB and gabapentin share several advantages such as a lack of pharmacokinetic drug interactions and similar mechanism of action and efficacy. It is unclear if PGB has any clinical advantage over gabapentin, though, as there have been no direct comparisons done in clinical trials between the two drugs. ${ }^{24}$ Unlike gabapentin, PGB exhibits linear pharmacokinetics following oral administration with low intersubject variability, which results in a more predictable dose-response relationship, since plasma concentrations increase linearly with increasing dose. Additionally, because of the pharmacokinetic advantages PGB has over gabapentin, patients can be initiated and titrated up to a target dose more rapidly. A dose-response relationship was demonstrated in two of the PGB trials discussed. ${ }^{7,18}$ Based on clinical trial data presented here and recommendations in $\mathrm{PGB}$ product labeling, patients may see more improvements at higher doses and should be titrated up to $600 \mathrm{mg} /$ day if they do not experience adequate relief at lower doses and can tolerate the higher PGB doses. ${ }^{7,18,20}$ Gabapentin has a cost advantage over PGB since gabapentin has a lower cost generic available, but the monthly cost of PGB is similar to generic gabapentin at the highest target doses of gabapentin. ${ }^{24,28}$ The long-term economic impact of PGB in treating PHN is unclear. ${ }^{24}$

A recently-updated meta-analysis on treatments for all types of neuropathic pain provides an evidence-based treatment algorithm for peripheral neuropathic pain. $\mathrm{PGB} /$ gabapentin and 
TCAs/selective serotonin-norepinephrine reuptake inhibitors (SNRIs) were considered essentially equivalent as drugs of first choice based on pooled NNT and number needed to harm (NNH) data from available RCTs. ${ }^{29}$ SNRIs show promise as potential therapies for other types of neuropathic pain, but clinical trial data to support their use for PHN are not yet available. ${ }^{29,30}$ PGB and gabapentin were treated as essentially "interchangeable" since the authors felt that the data were insufficient to allow for separate analyses of PGB and gabapentin. ${ }^{19,29}$ If a patient has a contraindication to using a TCA, such as ischemic heart disease or cardiac conduction abnormalities, PGB/gabapentin would be preferred over a TCA. ${ }^{10,29}$ A subsequent cost-effectiveness analysis by Smith and Roberts ${ }^{30}$ largely agreed with the algorithm recommended by Finnerup et $\mathrm{al}^{29}$ suggesting that gabapentin/PGB and TCAs appeared to be reasonable first choices for PHN from both a clinical and economic standpoint in patients without coronary artery disease, while gabapentin/PGB were clearly favored in patients with coronary artery disease or who had other contraindications to using TCAs. ${ }^{30}$ Smith and Roberts also examined PGB and gabapentin data separately, and, they noted that, if treated separately, gabapentin appeared to be preferred over PGB due to higher side effect discontinuance rates in some trials. ${ }^{30}$ Another cost-effective analysis comparing desipramine, gabapentin, and PGB for the treatment of PHN in hypothetical older adults 60 to 80 years of age concluded that desipramine was more cost-effective than the other agents. ${ }^{31}$ However, the study only analyzed the use of these three agents in patients with no known ischemic heart disease or no cardiac conduction abnormalities.

It is important to note that many $\mathrm{PHN}$ patients require trials of different therapies to achieve adequate pain relief; in fact, they often require more than 1 agent to manage their pain. There is no clinical trial or cost-effectiveness data available on PGB used in combination therapy for PHN.

\section{Conclusion}

$\mathrm{PHN}$ is a devastating consequence of $\mathrm{HZ}$ that significantly affects patient quality of life. Despite positive findings with several drug classes, the very heterogeneous nature of PHN makes successful pain management difficult. The response to therapy may vary within a single patient as well as from patient to patient. Often, trials of more than 1 agent are necessary before adequate pain management is achieved. Regardless of which agent is tried first, due to the complexity of PHN symptoms, it is not surprising that a single agent may not provide adequate pain relief. Many patients require a combination of therapies each ideally targeting different pain mechanisms. Ultimately, though, each pain management regimen needs to be tailored to the individual patient.

PGB appears to be an efficacious, well-tolerated option for the treatment of PHN. PGB has some advantages over other available, recommended therapies, but cost may be an issue versus other established treatments. Additionally, long-term safety and efficacy of PGB still need to be established.

\section{Disclosures}

The author has no conflicts of interest to declare.

\section{References}

1. Bader MS, McKinsey DS. Viral infections in the elderly: the challenges of managing herpes zoster, influenza, and RSV. Postgrad Med. 2005; $118: 45-54$

2. Stacey BR, Glanzman RL. Use of gabapentin for postherpetic neuralgia: results of two randomized, placebo-controlled studies. Clin Ther. 2003;25:2597-2608.

3. Argoff CE, Katz N, Backonja M. Treatment of Postherpetic Neuralgia: A Review of Therapeutic Options. J Pain Symptom Manage. 2004;28:396-411.

4. Mounsey AL, Matthew LG, Slawson DC. Herpes zoster and postherpetic neuralgia: prevention and management. Am Fam Physician. 2005; 72:1075-1080.

5. Dubinsky RM, Kabbani H, El-Chami Z, et al. Practice parameter: treatment of postherpetic neuralgia: an evidence-based report of the Quality Standards Subcommittee of the American Academy of Neurology. Neurology. 2004;63:959-965.

6. Johnson RW. Pain following herpes zoster: implications for management. Herpes. 2004;11:63-65.

7. Sabatowski R, Galvez R, Cherry DA, et al. Pregabalin reduces pain and improves sleep and mood disturbances in patients with post-herpetic neuralgia: results of a randomised, placebo-controlled clinical trial. Pain. 2004;109:26-35.

8. Engberg IB, Gröndahl GB, Thibom K. Patients' experiences of herpes zoster and postherpetic neuralgia. $J$ Adv Nurs. 1995;21:427-433.

9. Moulin DE, Clark AJ, Gilron MD, et al. Pharmacological management of chronic neuropathic pain - Consensus statement and guidelines from the Canadian Pain Society. Pain Res Manage. 2007;12:13-21.

10. Dworkin RH, Miroslav B, Rowbotham C, et al. Advances in neuropathic pain: diagnosis, mechanisms, and treatment recommendations. Arch Neurol. 2003;60:1524-1534.

11. Attal N, Cruccu G, Haanpää M, et al. EFNS guidelines on pharmacological treatment of neuropathic pain. Eur J Neurol. 2006;13:1153-1169.

12. Gore M, Sadosky A, Tai Kei-Sing, et al. A retrospective evaluation of the use of gabapentin and pregabalin in patients with postherpetic neuralgia in usual-care settings. Clin Ther. 2007;29:1655-1670.

13. Finnerup NB, Otto M, Jensen TS, et al. An evidence-based algorithm for the treatment of neuropathic pain. MedGenMed. 2007;9:36.

14. Dworkin RH, Corbin AE, Young JP, et al. Pregabalin for the treatment of postherpetic neuralgia: a randomized, placebo-controlled trial. Neurology. 2003;60:1274-1283.

15. Bockbrader HN, Burger P, Corrigan BW. Population pharmacokinetics of pregabalin in healthy volunteers, renally impaired patients, and patients with chronic pain. Presented at the meeting of the American Pain Society; March 14-17, 2002;Baltimore, MD.

16. Rowbotham M, Harden N, Stacey B, et al. Gabapentin for the treatment of postherpetic neuralgia: a randomized controlled trial. JAMA. 1998;280:1837-1842.

17. Rice ASC, Maton S, Postherpetic Neuralgia Study Group. Gabapentin in postherpetic neuralgia: a randomised, double blind, placebo controlled study. Pain. 2001;94:215-224. 
18. Van Seventer R, Feister HA, Young JP, et al. Efficacy and tolerability of twice-daily pregabalin for treating pain and related sleep interference in postherpetic neuralgia: a 13-week, randomized trial. Curr Med Res Opin. 2006;22:375-384.

19. Guay DRP. Pregablin in neuropathic pain: a more "pharmaceutically elegant" gabapentin? Am J Geriatr Pharmacother. 2005;3:274-287.

20. Lyrica $^{\circledR}$ [package insert]. New York: Pfizer Inc; June 2007.

21. Cada DJ, Levien T, Baker DE. Pregabalin. Hospital Pharmacy. 2006;41:157-172.

22. Frampton JE, Foster RH. Pregabalin in the treatment of postherpetic neuralgia. Drugs. 2005;65:111-118.

23. Neurontin [package insert]. New York: Pfizer Inc; January 2007.

24. Blommel ML, Blommel AL. Pregabalin: an antiepileptic agent useful for neuropathic pain. Am J Health-Syst Pharm. 2007;64:1475-1482.

25. Freynhagen R, Strojek K, Griesing T, et al. Efficacy of pregabalin in neuropathic pain evaluated in a 12-week, randomised, double-blind, multicentre, placebo-controlled trial of flexible- and fixed-dose regimens. Pain. 2005;115:254-263.
26. Randinitis EJ, Posvar EL, Alvey CW, et al. Pharmacokinetics of pregabalin in subjects with various degrees of renal function. $J$ Clin Pharmacol. 2003;43:277-283.

27. Murphy N, Mockler M, Ryder M, et al. Decompensation of chronic heart failure associated with pregabalin in patients with neuropathic pain. J Card Fail. 2007;13:227-229.

28. Drugstore.com [homepage on the Internet]. Drugstore.com, Inc; c1999-2008 [cited 2008 Sept 4]. Available from: http://drugstore.com.

29. Finnerup NB, Otto M, McQuay HJ. Algorithm for neuropathic pain treatment: an evidence based proposal. Pain. 2005;118:289-305.

30. Smith KJ, Roberts MS. Sequential medication strategies for postherpetic neuralgia: a cost-effectiveness analysis. J Pain. 2007;8:396-404.

31. O'Connor AB, Noyes K, Holloway RG. A cost-effectiveness comparison of desipramine, gabapentin, and pregabalin for treating postherpetic neuralgia. J Am Geriatr Soc. 2007;55:1176-1184. 
